УДК 624.078

\section{О.А. Ковальчук}

\section{МОДЕЛИРОВАНИЕ ПРОСТРАНСТВЕННЫХ СТЕРЖНЕВЫХ СИСТЕМ МЕТОДОМ КОНЕЧНЫХ ЭЛЕМЕНТОВ}

Рассмотрено представление конструкции каркасного здания пространственной стержневой системой и моделирование полученной стержневой системы методом конечных элементов. Составлены уравнения состояния стержня и получено решение системы уравнений в форме Коши. Получена матрица жесткости, связывающая узловые силы и узловые перемещения. Полученные результаты применимы как для статически определимых, так и для статически неопределимых систем.

Ключевые слова: пространственная стержневая система, метод конечных элементов (МКЭ), метод перемещений, узловое перемещение, вектор узловых перемещений, вектор узловых сил, матрица жесткости.
O.A. Kovalchuk

\section{THE SIMULATION OF THREE-DIMENSIONAL BAR SYSTEMS BY THE FINITE ELEMENT METHOD}

The article considers the representation of framed building as a three-dimensional bar system and simulation of obtained bar system by the finite element method. Underway the bar state equations and the equations system solution in the Cauchy form were obtained. Also article deals with obtaining of stiffness matrix linking nodal forces and nodal displacements. The obtained results are applicable for both the statically determinate systems and the statically indeterminate ones.

Key words: three-dimensional bar system, finite element method, deflection method, node displacement, node displacement vector, node force vector, stiffness matrix.

Конструкции каркасных зданий могут быть представлены системой прямых стержней. Наиболее распространен для их моделирования метод конечных элементов (МКЭ), который позволяет найти состояние каждого стержня путем разбиения каждого стержня на конечные элементы (КЭ) стержни меньшей длины, причем при увеличении количества КЭ решение стремится к точному в рамках принятой математической модели. Рассмотрим один из возможных приемов формулировки соотношений для КЭ, считая, что материал линейно-упругий, деформации малы, малы углы поворота сечения, справедливы гипотезы плоских сечений и ненадавливания слоев, параллельных оси, друг на друга [1-3]. Примем систему декартовых координат $x, y, z$, где $x$ - продольная (осевая) координата, $y, z$ - главные центральные оси инерции. Будем считать, что оси $x, y, z$ совмещены с ортами естественного трехгранника (трехгранника Френе $[3,4]) t, n, b$ (касательной, нормалью, бинормалью).

В рамках этих гипотез уравнения состояния стержня могут быть записаны в виде (1), где $u, v, w-$ компоненты вектора перемещения в выбранной системе координат; $\vartheta$, $\vartheta_{b}, \vartheta_{n}-$ углы поворота сечения относительно ортов естественного трехгранника; $M_{t}$ - крутящий, $M_{b}$, $M_{n}$ - изгибающие моменты; $N$ - осевая (тангенциальная); $Q_{n}, Q_{b}$ - поперечные силы, первая (левая) группа уравнений есть геометрические и фи-

$$
\begin{aligned}
& \frac{\partial u}{\partial x}=\frac{N}{E A} ; \frac{\partial M_{\mathrm{t}}}{\partial x}=\ddot{\theta}_{t} J_{t} \rho ; \\
& \frac{\partial v}{\partial x}=\theta_{b} ; \frac{\partial M_{b}}{\partial x}=Q_{n} ; \\
& \frac{\partial w}{\partial x}=\theta_{n} ; \frac{\partial M_{b}}{\partial x}=Q_{n} ; \\
& \frac{\partial \theta_{t}}{\partial x}=\frac{M_{t}}{G J_{t}} ; \frac{\partial N}{\partial x}=\ddot{u} \rho A-q_{t} ; \\
& \frac{\partial \theta_{b}}{\partial x}=-\frac{M_{b}}{E J_{b}} ; \frac{\partial Q_{n}}{\partial x}=\ddot{v} \rho A-q_{n} ; \\
& \frac{\partial \theta_{n}}{\partial x}=-\frac{M_{n}}{E J_{n}} ; \frac{\partial Q_{b}}{\partial x}=\ddot{w} \rho A-q_{b} .
\end{aligned}
$$


зические уравнения, правая группа - уравнения движения. Инерция поворота сечения относительно ортов $n$ и $b$ не учитывается.

Вводя вектор состояния

$$
y(x, t)=\left\{\begin{array}{llllllllllll}
u & v & w & \theta_{t} & \theta_{b} & \theta_{n} & M_{t} & M_{b} & M_{n} & N & Q_{n} & Q_{b}
\end{array}\right\}(x, t),
$$

перепишем (1) в матричной форме:

$$
y^{\prime}=A y+M \ddot{y}-q(x, t),
$$

где ненулевые компоненты матриц $A$ и $M$ очевидным образом определяются из уравнений состояния (2),

$$
q(x, t)=\left\{\begin{array}{llllllllllll}
0 & 0 & 0 & 0 & 0 & 0 & 0 & 0 & 0 & q_{t} & q_{n} & q_{t}
\end{array}\right\}(x, t) .
$$

Рассмотрим пока задачу статики, опуская инерционные члены (полагая М нулевой матрицей). Тогда из (3) получим:

$$
y^{\prime}=A y-q(x) .
$$

Это система обыкновенных дифференциальных уравнений с постоянной матрицей. Ее решение примем в форме Коши [2]:

$$
y(x)=V(x) y_{0}+F_{q}(x), V(0)=I_{12},
$$

где $I_{12}$ - единичная матрица 12 порядка; $y_{0}$ - состояние стержня в начале координат (начальные параметры); $V(x)$ - матрица фундаментальных решений $(5) ; F_{q}(x)$ - частное решение (5), соответствующее заданным распределенным нагрузкам (4). Найти $V(x)$ и $F_{q}(x)$ можно, применяя к (5) преобразование Лапласа:

$$
\begin{aligned}
& y^{*}(s)=V^{*}(s)\left\{y_{0}+q^{*}(s)\right\} \underset{\text { Обр. Лапл. } s}{\longrightarrow} y(x)=V(x) y_{0}+F_{q}(x), \\
& \left\{s I_{12}-A\right\}^{-1} \underset{\text { Обр. Лапл. } s}{\longrightarrow} V(x) ; \quad F_{q}(x)=\int_{0}^{x} V(x-\xi) q(\xi) d \xi .
\end{aligned}
$$

Здесь использована теорема о свертке [5]. Таким образом, состояние в любой точке стержня выражается через начальные параметры $\mathbf{y}_{0}$.

Используем форму решения (6). Предварительно отметим следующее: вектор состояния (2) можно представить в виде разбиения на два подвектора, один из которых содержит в себе только кинематические, второй - только силовые факторы:

$$
\begin{aligned}
& y=\left\{\begin{array}{ll}
y_{C} & y_{F}
\end{array}\right\}, \\
& y_{C}=\left\{\begin{array}{lllllll}
u & v & w & \theta_{t} & \theta_{b} & \theta_{n}
\end{array}\right\}, \\
& y_{F}=\left\{\begin{array}{llllllll}
M_{t} & M_{b} & M_{n} & N & Q_{n} & Q_{b}
\end{array}\right\} .
\end{aligned}
$$

Тогда (6) можно представить разбиением

$$
\begin{aligned}
& y_{C}(x)=V_{C C}(x) y_{0 C}+V_{C F}(x) y_{0 F}+F_{q C}(x), \\
& y_{F}(x)=V_{F C}(x) y_{0 C}+V_{F F}(x) y_{0 F}+F_{q F}(x) .
\end{aligned}
$$

Исключим силовые факторы в начале стержня через перемещения его конца, записывая первое уравнение (9) для конца стержня при $s=L$ :

$$
\begin{aligned}
& y_{C}(L)=y_{k C}=V_{C C}(L) y_{0 C}+V_{C F}(L) y_{0 F}+F_{q C}(L) ; \Rightarrow \\
& y_{0 F}=V_{C F}(L)^{-1}\left\{y_{k C}-V_{C C}(L) y_{0 F}-F_{q C}(L)\right\} .
\end{aligned}
$$

Теперь подставим результат в (9) и получим выражения для состояния в любой точке стержня через узловые перемещения: 


$$
\begin{aligned}
y_{C}(x)= & \left\{V_{C C}(x)-V_{C F}(x) V_{C F}(L)^{-1} V_{C C}(L)\right\} y_{0 C}+V_{C F}(x) V_{C F}(L)^{-1} y_{k C}+F_{q C}(x)- \\
& -V_{C F}(x) V_{C F}(L)^{-1} F_{q C}(L) ; \\
y_{F}(x)= & \left\{V_{F C}(x)-V_{F F}(x) V_{C F}(L)^{-1} V_{C C}(L)\right\} y_{0 C}+V_{F F}(x) V_{C F}(L)^{-1} y_{k C}+F_{q F}(x)- \\
& -F_{F F}(x) V_{C F}(L)^{-1} F_{q C}(L) .
\end{aligned}
$$

Введем вектор узловых перемещений так:

$$
u=\left\{y_{0 C} \quad y_{k C}\right\} .
$$

Тогда выражение для вектора состояния примет вид

$$
y(x)=D(s) u+F_{q 1}(x),
$$

где

$$
\begin{aligned}
& D(x)=\left[\begin{array}{ll}
V_{C C}(x)-V_{C F}(x) V_{C F}(L)^{-1} V_{C C}(L) & V_{C F}(x) V_{C F}(L)^{-1} \\
V_{F C}(s)-V_{F F}(s) V_{C F}(L)^{-1} V_{C C}(L) & V_{F F}(s) V_{C F}(L)^{-1}
\end{array}\right] ; \\
& F_{q 1}(x)=\left\{\begin{array}{l}
F_{q C}(x)-V_{C F}(x) V_{C F}(L)^{-1} F_{q C}(L) \\
F_{q F}(x)-V_{F F}(x) V_{C F}(L)^{-1} F_{q C}(L)
\end{array}\right\} .
\end{aligned}
$$

Теперь запишем выражение узловых сил через узловые перемещения, записывая первое уравнение (11) и последнее оттуда же, но при $x=L$ :

$$
\begin{aligned}
& y_{0 F}=V_{C F}(L)^{-1} y_{k C}-V_{C F}(L)^{-1} V_{C F}(L) y_{0 F}-V_{C F}(L)^{-1} F_{q C}(L), \\
& y_{k F}(L)=\left\{V_{F C}(L)-V_{F F}(L) V_{C F}(L)^{-1} V_{C F}(L)\right\} y_{0 C}+V_{F F}(L) V_{C F}(L)^{-1} y_{k C}+ \\
&+F_{q F}(L)-V_{F F}(L) V_{C F}(L)^{-1} F_{q C}(L) .
\end{aligned}
$$

Можно придать последним выражениям следующий вид:

$$
F=K u+F_{q 2},
$$

где

$$
\begin{aligned}
& F=\left\{\begin{array}{ll}
y_{0 F} & y_{k F}
\end{array} ; \quad F_{q 2}=\left\{\begin{array}{c}
-V_{C F}(L)^{-1} F_{q C}(L) \\
F_{q F}(L)-V_{F F}(L) V_{C F}(L)^{-1} F_{q C}(L)
\end{array}\right\} ;\right. \\
& K=\left[\begin{array}{cc}
-V_{C F}(L)^{-1} V_{C C}(L) y_{0 F} & V_{C F}(L)^{-1} \\
V_{F C}(L)-V_{F F}(L) V_{C F}(L)^{-1} V_{C C}(L) & V_{F F}(L) V_{C F}(L)^{-1}
\end{array}\right] .
\end{aligned}
$$

Выражение (15) дает нам сумму внешних (второе слагаемое) и внутренних сил (включая и силы инерции), сходящихся в одном узле. Тогда из него получаем разрешающее уравнение для одного стержня:

$$
K u+F_{q 2}=0 .
$$

Матрица $K$ связывает узловые силы и узловые перемещения и поэтому может быть названа матрицей жесткости, а вектор $F_{q 2}$ - вектором узловых сил, эквивалентным распределенной нагрузке. Выражение (18) по форме такое же, как и аналогичное уравнение МКЭ, и, следовательно, к нему можно применить известный алгоритм МКЭ в форме метода перемещений.

Так как уравнение (18) по форме аналогично разрешающему уравнению МКЭ, то для моделирования стержневой системы можно использовать традиционный алгоритм метода перемещений МКЭ [6, 7].

Далее приводятся решения некоторых простых задач для одного стержня и стержневых систем, которые поддаются проверке методом сечений. Материал стержней - сталь, размеры определялись глобальными координатами узлов, поперечное сечение - прямоугольник с соотношением сторон высота : ширина $=2: 1$. Вместо эпюр внутренних силовых и кинематических факторов для каждого стержня приведены их графики в локальных координатах, а также формы деформированной стержневой системы. При- 
нято, что вектор нормали лежит в вертикальной плоскости, включающей в себя ось стержня.
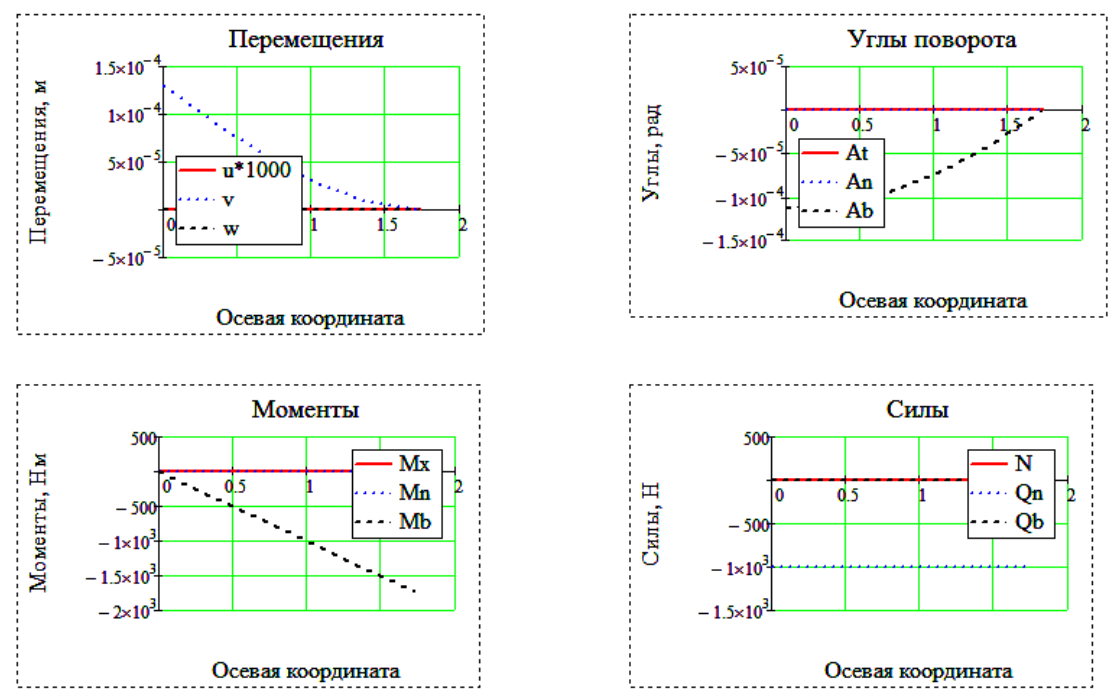

Рис. 1. Консольный стержень, нагруженный силой в начале, действующей по нормали к оси

Стойка - стержень 0-1

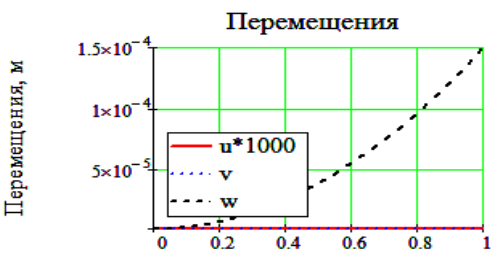

Осевая координата

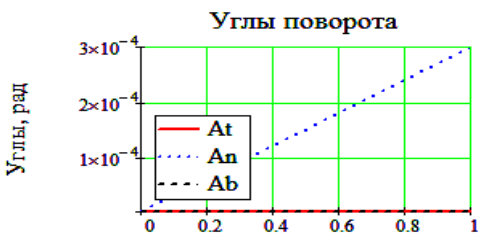

Осевая координата

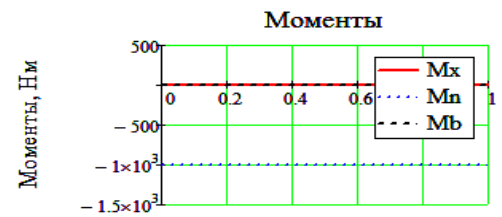

Осевая координата

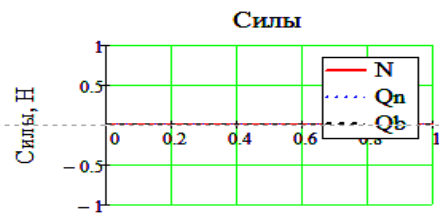

Осевая координата
Ригель - стержень 1-2

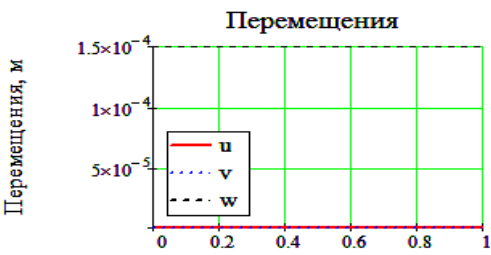

Осевая координата

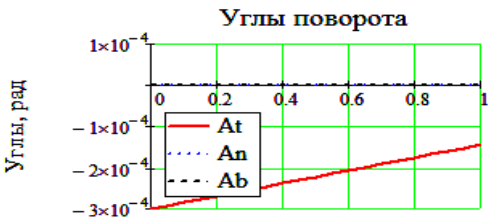

Oсевая координата

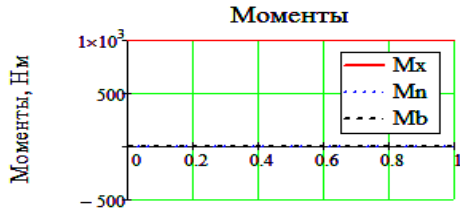

Осевая координата

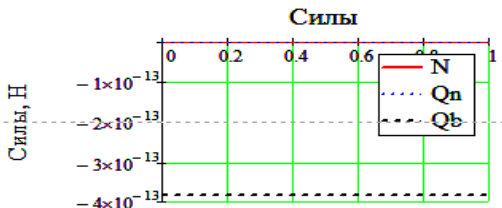

Осевая координата

Рис. 2. Плоская Г-образная рама, защемленная одним концом и нагруженная крутящим моментом на другом 


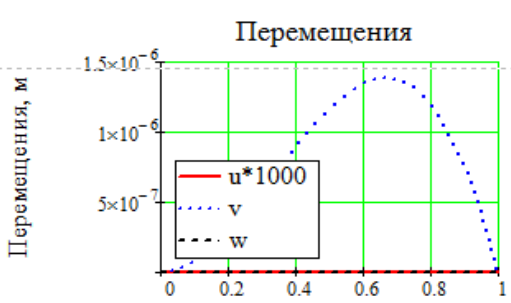

Осевая координата

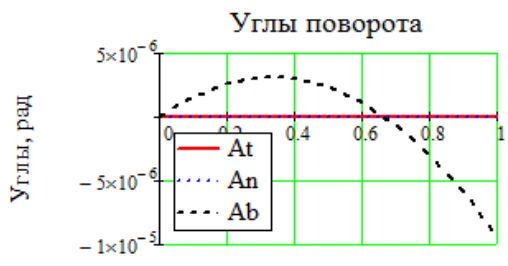

Осевая координата

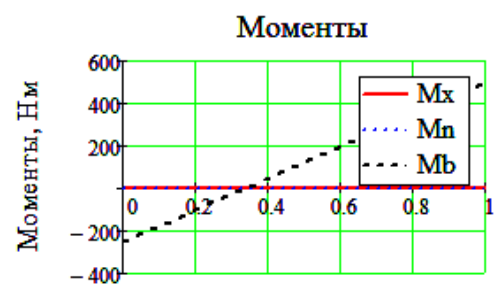

Осевая координата

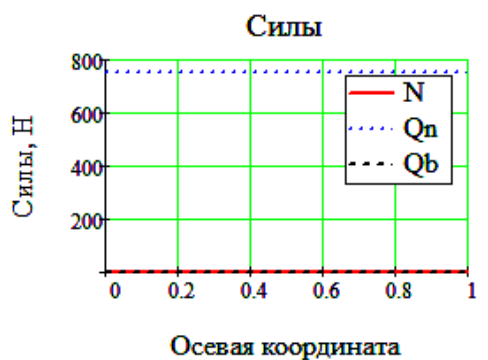

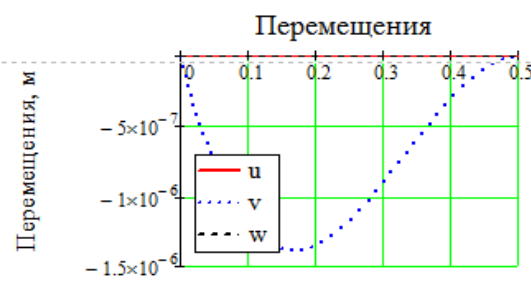

Осевая координата

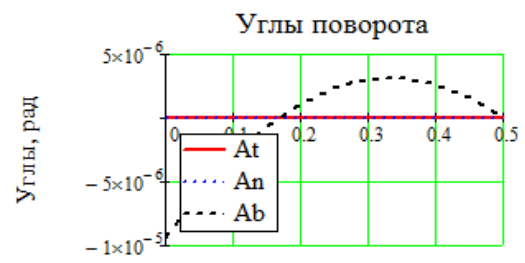

Осевая координата

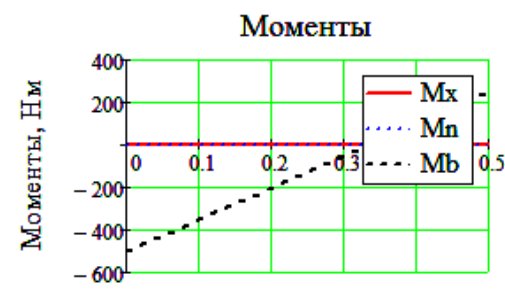

Осевая координата

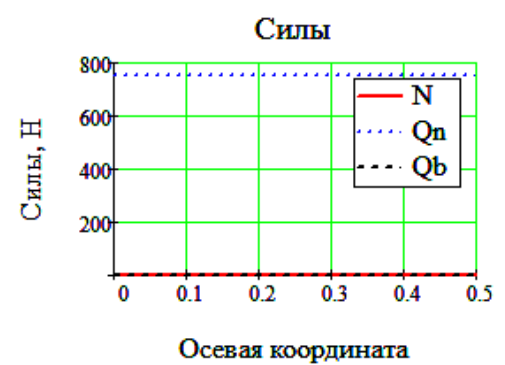

Рис. 3. Стержень, защемленный двумя концами, нагруженный моментом посередине

Результаты, приведенные на рис. 1, 2, 3 [8], не противоречат кинематическим и статическим воззрениям на состояния стержней и их систем. Расчеты возможны как для статически определимых, так и для статически неопределимых систем, что определяется применением алгоритма МКЭ для создания ансамбля конечных элементов.

\section{БИБЛИОГРАФИЧЕСКИЙ СПИСОК}

1. Александров А.В., Лащчеников Б.Я., Шапошников Н.Н. Строительная механика. Тонкостенные пространственные системы. М. : Стройиздат, 1983. 488 с.

2. Александров А.В., Потапов В.Д., Державин Б.П. Сопротивление материалов. М. : Высшая школа, 2003. 560 с.

3. Толоконников Л.А. Механика деформируемого твердого тела. М. : Высшая школа, 1979. $318 \mathrm{c}$.

4. Корн Г.А., Корн Т.М. Справочник по математике. М. : Наука, 1978.720 с.

5. Шостак Р.Я. Операционное исчисление. М. : Высшая школа, 1972. 279 с.

6. Образиов И.Ф., Савельев Л.М., Хазанов Х.С. Метод конечных элементов в задачах строительной механики летательных аппаратов. М. : Высшая школа, 1985. 392 с. 
7. Постнов В.А., Хархурим И.Я. Метод конечных элементов в расчетах судовых конструкций. Л. : Судостроение, 1974. 476 с.

8. Макаров Е.Г. Сопротивление материалов на базе Mathcad. СПб : БХВ-Петербург, 2004. 512 c.

\section{REFERENCES}

1. Aleksandrov A.V., Lashchenikov B.Ja., Shaposhnikov N.N. Stroitel'naya mekhanika. Tonkostennye prostranstvennye sistemy [Structural Mechanics. Thin-walled three-dimensional system]. Moscow, 1983, p. 488.

2. Aleksandrov A.V., Potapov V.D., Derzhavin B.P. Soprotivlenie materialov [Strength of the materials]. Moscow, 2003, p. 560.

3. Tolokonnikov L.A. Mehanika deformiruemogo tverdogo tela [Deformable solid mechanics]. Moscow, 1979. p. 318.

4. Korn G.A., Korn T.M. Spravochnik po matematike [References book of Mathematics]. Moscow, 1978. p. 720.

5. Shostak R.Ja. Operatsionnoe ischislenie [Operational calculus]. Moscow, 1972, p. 279.

6. Obraztsov I.F., Savel'ev L.M., Hazanov X.S. Metod konechnykh elementov v zadachakh stroitel'noy mekhaniki letatel'nykh apparatov [The finite element method for the problems of aircraft structural theory]. Moscow, 1985, p. 392.

7. Postnov V.A., Harhurim I.Ja. Metod konechnykh elementov v raschetahh sudovyhh konstruktsiy [The finite element method for the ship structures calculation], Leningrad, 1974, p. 476.

8. Makarov E.G. Soprotivlenie materialov na baze Mathcad [Constructional materials based on Mathcad]. St. Petersburg, BHV, 2004, p. 512.

Поступила в редакцию в апреле 2012 г

Об авторе: Ковальчук Олег Александрович, кандидат технических наук, доцент, директор Института фундаментального образования, ФГБОУ ВПО «МГСУ», 129337, г. Москва, Ярославское шоссе, д. 26, oko44@mail.ru
About the author: Kovalchuk Oleg Aleksandrovich candidate of technical sciences, docent, director of the Fundamental Education Institute, Moscow State University of Civil Engineering (MSUCE), 26 Yaroslavskoye shosse, 129337, Moscow, Russian Federation, oko44@mail.ru

Для цитирования:

Ковальчук О.А. Моделирование пространственных стержневых систем методом конечных элементов // Научно-практический Интернет-журнал «Наука. Строительство. Образование». 2012. Вып. 2. Режим доступа: http://www.nso-journal.ru.

For citation:

Kovalchuk O.A. Modelirovanie prostranstvennykh sterzhnevykh system metodom konechnykh elementov [The simulation of three-dimensional bar systems by the finite element method] Nauchnoprakticheskiy Internet-zhurnal «Nauka. Stroitel'stvo. Obrazovanie» [Science, construction, education], 2012, no. 2. Available at: http://www.nso-journal.ru. 